Notre Dame Journal of Formal Logic

Volume 27, Number 4, October 1986

\title{
Sums of Finitely Many Ordinals of Various Kinds
}

\author{
MARTIN M. ZUCKERMAN
}

Abstract The ordinals $\alpha_{1}, \alpha_{2}, \ldots, \alpha_{n}$ are said to be pairwise-noncommutative if for all $i, j=1,2, \ldots, n$, if $i \neq j$, then $\alpha_{i}+\alpha_{j} \neq \alpha_{j}+\alpha_{i}$. For positive integers $n$ and $k$, let $\Sigma_{n}$ be the symmetric group on $n$ letters and let $E_{n}$ (respectively $L_{n}, S_{n}, T_{n}$, or $P_{n}$ ) be the set of all $k$ for which there exist $n$ (not necessarily distinct) nonzero ordinals (respectively, limit ordinals, successor ordinals, infinite successor ordinals, or pairwise-noncommutative ordinals) such that $\sum_{i=1}^{n} \alpha_{\phi(i)}$ takes on exactly $k$ values as $\phi$ ranges over $\Sigma_{n}$. Then for all $n \geq 1, E_{n}=L_{n}=$ $S_{n}=T_{n} ; \min P_{n}=n$, and $\max P_{n}=\max E_{n}$. Furthermore, $P_{1}=E_{1}, P_{2}=E_{2}$, $P_{3}=E_{3}-\{1,2\}$, and $P_{4}=E_{4}-\{1,2,3,11\}$.

1 Introduction Addition of ordinal numbers depends upon the order of the summands. For each positive integer $n$, the maximum number, $m_{n}$, of distinct values that can be assumed by a sum of $n$ nonzero ordinal numbers in all $n$ ! permutations of the summands has been calculated by Erdös [1] and Wakulicz [3] and [4]. The first few values of $m_{n}$ are as follows: $m_{1}=1, m_{2}=2, m_{3}=5$, $m_{4}=13, m_{5}=33, m_{6}=81, m_{7}=193, m_{8}=449$; moreover, it is known that $\lim _{n \rightarrow \infty} \frac{m_{n}}{n !}=0$.

Let $n$ and $k$ be positive integers. Let $\Sigma_{n}$ be the symmetric group on $n$ letters. Let $\alpha_{1}, \alpha_{2}, \ldots, \alpha_{n}$ be any $n$ (not necessarily distinct) nonzero ordinals. We will say that $\alpha_{1}, \alpha_{2}, \ldots, \alpha_{n}$ yield $k$ sums if $\left\{\sum_{i=1}^{n} \alpha_{\phi(i)}: \phi \in \Sigma_{n}\right\}$ is a $k$-element set. Let $E_{n}$ be the set of all integers $k$ for which there exist $n$ (not necessarily distinct) nonzero ordinals that yield $k$ sums. It is known that $E_{n}=\{1,2,3$, $\left.\ldots, m_{n}\right\}$ for $n=1,2,3,4,6,7$, and 8 ([2], [5], and [6]), that $E_{5}=\{1,2,3, \ldots$, $29\} \cup\{31,32,33\}([3])$, and that $E_{n}$ is properly included in $\left\{1,2,3, \ldots, m_{n}\right\}$ for all $n \geq 9$ ([7]).

For every ordinal number $\alpha>0$, let

(1) $\alpha=\omega^{\lambda_{1}} a_{1}+\omega^{\lambda_{2}} a_{2}+\ldots+\omega^{\lambda_{r}} a_{r}$ 
be the (Cantor) normal form of $\alpha$; here $r, a_{1}, a_{2}, \ldots, a_{r}$ are positive integers and $\lambda_{1}>\lambda_{2}>\ldots>\lambda_{r} \geq 0$ are ordinals. $\lambda_{1}$ is called the degree of $\alpha$ (written, "deg $\alpha$ ") and $\alpha_{1}$, the leading coefficient of $\alpha$. By the remainder of $\alpha$, we mean $\omega^{\lambda_{2}} a_{2}+\ldots+\omega^{\lambda_{r}} a_{r}$ (or zero, if $r=1$ ). By the remainder form of $\alpha$, we mean $\omega^{\lambda_{1}} a_{1}+\rho_{1}$, where $\lambda_{1}$ is the degree of $\alpha, a_{1}$ is the leading coefficient of $\alpha$, and $\rho_{1}$ is the remainder of $\alpha$.

The ordinal numbers $\alpha_{1}, \alpha_{2}, \ldots, \alpha_{n}$ are said to be nonoverlapping if for each $i, j(\neq i)=1,2, \ldots, n$, whenever $\lambda_{i}=\operatorname{deg} \alpha_{i}>\operatorname{deg} \alpha_{j}=\lambda_{j}$, then (1), the normal form of $\alpha_{i}$, consists of terms all of which are of degree $>\lambda_{j}$. Addition of nonoverlapping ordinals is considerably simpler than in the general case, and is considered in [6]. Here and in [8] we consider the addition of various other types of ordinals.

2 Limit ordinals; successor ordinals Let $L_{n}$ be the set of all integers $k$ for which there exist $n$ (not necessarily distinct) limit ordinals that yield $k$ sums; let $S_{n}$ be the set of all integers $k$ for which there exist $n$ (not necessarily distinct) successor ordinals that yield $k$ sums, and let $T_{n}$ be the set of all integers $k$ for which there exist $k$ (not necessarily distinct) infinite successor ordinals that yield $k$ sums.

Theorem 1 For all $n=1,2,3, \ldots, E_{n}=L_{n}=S_{n}=T_{n}$.

Proof: Clearly, $L_{n} \subseteq E_{n}$ and $T_{n} \subseteq S_{n} \subseteq E_{n}$.

For any nonzero ordinal $\alpha$ whose normal form is given by (1), let

$$
\begin{aligned}
& \alpha^{\prime}=\omega^{\lambda_{1}+1} a_{1}+\omega^{\lambda_{2}+1} a_{2}+\ldots+\omega^{\lambda_{r}+1} a_{r} \\
& \alpha^{\prime \prime}= \begin{cases}\alpha+1, \text { if } \alpha \text { is infinite } \\
\alpha, \text { if } \alpha \text { is finite }\end{cases}
\end{aligned}
$$

and

$$
\alpha^{\prime \prime \prime}=\omega^{\lambda_{1}+1} a_{1}+\omega^{\lambda_{2}+1} a_{2}+\ldots+\omega^{\lambda_{r}+1} a_{r}+1 .
$$

Let $k \in E_{n}$ and let $\alpha_{1}, \alpha_{2}, \ldots, \alpha_{n}$ yield $k$ sums. Suppose that for some $\phi \in \Sigma_{n}$,

$$
\sum_{i=1}^{n} \alpha_{\phi(i)}=\omega^{\delta_{1}} b_{1}+\omega^{\delta_{2}} b_{2}+\ldots+\omega^{\delta_{s}} b_{s} .
$$

Then

$$
\sum_{i=1}^{n}\left(\alpha_{\phi(i)}{ }^{\prime}\right)=\omega^{\delta_{1}+1} b_{1}+\omega^{\delta_{2}+1} b_{2}+\ldots+\omega^{\delta_{s}+1} b_{s}
$$

so that $\alpha_{1}{ }^{\prime}, \alpha_{2}{ }^{\prime}, \ldots, \alpha_{n}{ }^{\prime}$ yield $k$ sums, and consequently, $E_{n} \subseteq L_{n}$. Clearly $1 \in$ $S_{n}$ for all $n$. To see that $E_{n} \subseteq S_{n}$ for all $n$, we can assume that at least one of the ordinals $\alpha_{1}, \alpha_{2}, \ldots, \alpha_{n}$ is infinite. Then

$$
\sum_{i=1}^{n}\left(\alpha_{\phi(i)^{\prime \prime}}\right)=\left(\sum_{i=1}^{n} \alpha_{\phi(i)}\right)+1
$$

so that $\alpha_{1}^{\prime \prime}, \alpha_{2}^{\prime \prime}, \ldots, \alpha_{n}{ }^{\prime \prime}$ yields $k$ sums, and $E_{n} \subseteq S_{n}$. Finally, 


$$
\sum_{i=1}^{n}\left(\alpha_{\phi(i)}^{\prime \prime \prime}\right)=\omega^{\delta_{1}+1} b_{1}+\omega^{\delta_{2}+1} b_{2}+\ldots+\omega^{\delta_{s}+1} b_{s}+1
$$

so that $\alpha_{1}^{\prime \prime \prime}, \alpha_{2}^{\prime \prime \prime}, \ldots, \alpha_{n}^{\prime \prime \prime}$ yield $k$ sums, and $E_{n} \subseteq T_{n}$. Thus for all $n, E_{n}=$ $L_{n}=S_{n}=T_{n}$, as was to be proved.

3 Pairwise-noncommutative ordinals Let $\alpha=\omega^{\lambda_{1}} a_{1}+\rho$ and $\beta=\omega^{\mu_{1}} b_{1}+\sigma$ be the remainder forms of the nonzero ordinals $\alpha$ and $\beta$, respectively. Then it is well-known that $\alpha+\beta=\beta+\alpha$ if and only if $\lambda_{1}=\mu_{1}$ and $\rho=\sigma$. In other words, two nonzero ordinals commute if and only if they agree in their degrees and in their remainders.

Let $\alpha_{1}, \alpha_{2}, \ldots, \alpha_{n}$ be any $n$ nonzero ordinals. Then $\alpha_{1}, \alpha_{2}, \ldots, \alpha_{n}$ are said to be pairwise-noncommutative if for all $i, j=1,2, \ldots, n$, if $i \neq j$, then $\alpha_{i}+$ $\alpha_{j} \neq \alpha_{j}+\alpha_{i}$. In many of the examples in [2], [3], [5], and [6], ordinals repeat, or more than one ordinal is finite, or several ordinals are integral multiples of $\omega$. These examples thus make use of $n$ ordinals, at least two of which commute. Addition of pairwise-noncommutative ordinals is considerably more restrictive.

For each $n$, let $P_{n}$ be the set of all integers $k$ for which there exist $n$ pairwise-noncommutative ordinals that yield $k$ sums.

Lemma 1 Suppose that for ordinals $\alpha$ and $\beta, \alpha+\beta \neq \beta+\alpha$.

If $\operatorname{deg} \beta<\operatorname{deg} \alpha$, then

$$
\alpha=\beta+\alpha<\alpha+\beta .
$$

If $\operatorname{deg} \beta=\operatorname{deg} \alpha$ and rem $\beta<\operatorname{rem} \alpha$, then

$$
\alpha+\beta<\beta+\alpha \text {. }
$$

Theorem 2 For all $n \geq 1, \min P_{n}=n$ and $\max P_{n}=m_{n}$.

Proof: We first show that for all $n \geq 1$, every set of $n$ pairwise-noncommutative ordinals yields at least $n$ distinct sums. For $n=1$, this is obvious.

Let $n>1$ and suppose that for $1 \leq k<n$, every set of $k$ pairwisenoncommutative ordinals yields at least $k$ distinct sums. Suppose that $\alpha_{1}$, $\alpha_{2}, \ldots, \alpha_{n}$ are pairwise-noncommutative ordinals and $\alpha_{1}<\alpha_{2}<\ldots<\alpha_{n}$. If $\operatorname{deg} \alpha_{n-1}<\operatorname{deg} \alpha_{n}$, let $A_{1}, A_{2}, \ldots, A_{n-1}$ be sums for $\alpha_{1}, \alpha_{2}, \ldots, \alpha_{n-1}$ such that $A_{1}<A_{2}<\ldots<A_{n-1}$. Then $\operatorname{deg} A_{1}<\operatorname{deg} \alpha_{n}$ so that, by Lemma 1,

$$
A_{1}+\alpha_{n}=\alpha_{n}<\alpha_{n}+A_{1}<\alpha_{n}+A_{2}<\ldots<\alpha_{n}+A_{n-1}
$$

and consequently,

$$
A_{1}+\alpha_{n}, \alpha_{n}+A_{1}, \alpha_{n}+A_{2}, \ldots, \alpha_{n}+A_{n-1}
$$

are $n$ distinct sums for $\alpha_{1}, \alpha_{2}, \ldots, \alpha_{n}$.

If $\operatorname{deg} \alpha_{n-1}=\operatorname{deg} \alpha_{n}$, let $m$ be the smallest index for which $\operatorname{deg} \alpha_{m}=$ $\operatorname{deg} \alpha_{n}$. If $m=1$ and if $A_{1}, A_{2}, \ldots, A_{n-1}$ are distinct sums for $\alpha_{1}, \alpha_{2}, \ldots, \alpha_{n-1}$ such that $A_{1}<A_{2}<\ldots<A_{n-1}$, then because $\alpha_{1}, \alpha_{2}, \ldots, \alpha_{n}, A_{1}, A_{2}, \ldots$, and $A_{n-1}$ are all of the same degree and because

$$
\begin{gathered}
\operatorname{rem} A_{1}=\operatorname{rem} \alpha_{1}<\operatorname{rem} \alpha_{2}=\operatorname{rem} A_{2}<\ldots< \\
\operatorname{rem} \alpha_{n-1}=\operatorname{rem} A_{n-1}<\operatorname{rem} \alpha_{n}
\end{gathered}
$$


it follows that

$$
\alpha_{n}+A_{1}<\alpha_{n}+A_{2}<\ldots<\alpha_{n}+A_{n-1}<A_{n-1}+\alpha_{n}
$$

so that $\alpha_{n}+A_{i}, i=1,2, \ldots, n-1$, together with $A_{n-1}+\alpha_{n}$ are $n$ distinct sums for $\alpha_{1}, \alpha_{2}, \ldots, \alpha_{n}$.

If $m>1$, then let

$$
\begin{aligned}
B_{m} & =\sum_{i=1}^{m-1} \alpha_{i}+\sum_{i=m+1}^{n-1} \alpha_{i}+\alpha_{m}=\sum_{i=m+1}^{n-1} \alpha_{i}+\alpha_{m} \\
B_{m+1} & =\sum_{i=1}^{m} \alpha_{i}+\sum_{i=m+2}^{n-1} \alpha_{i}+\alpha_{m+1}=\alpha_{m}+\sum_{i=m+2}^{n-1} \alpha_{i}+\alpha_{m+1} \\
\vdots & \\
B_{n-1} & =\sum_{i=1}^{n-1} \alpha_{i}=\sum_{i=m}^{n-1} \alpha_{i} .
\end{aligned}
$$

Then $B_{m}<B_{m+1}<\ldots<B_{n-1}$, so that $B_{m}, B_{m+1}, \ldots, B_{n-1}$ are $n-m$ distinct sums for $\alpha_{m}, \alpha_{m+1}, \ldots, \alpha_{n-1}$ as well as for $\alpha_{1}, \alpha_{2}, \ldots, \alpha_{n-1}$. Moreover,

(2) $\alpha_{n}+B_{m}<\alpha_{n}+B_{m+1}<\ldots<\alpha_{n}+B_{n-1}$

so that $\alpha_{n}+B_{i}, i=m, m+1, \ldots, n-1$, are $n-m$ distinct sums for $\alpha_{1}$, $\alpha_{2}, \ldots, \alpha_{n}$. Furthermore, by the inductive hypothesis, there are (at least) $m$ distinct sums, $C_{1}, C_{2}, \ldots, C_{m-1}, C_{n}$ for $\alpha_{1}, \alpha_{2}, \ldots, \alpha_{m-1}, \alpha_{n}$. We can assume that $C_{1}<C_{2}<\ldots<C_{m-1}<C_{n}$, and consequently,

$$
B_{n-1}+C_{1}<B_{n-1}+C_{2}<\ldots<B_{n-1}+C_{m-1}<B_{n-1}+C_{n} .
$$

Each of the ordinals $B_{n-1}+C_{i}, i=1,2, \ldots, m-1, n$, is a sum for $\alpha_{1}$, $\alpha_{2}, \ldots, \alpha_{n}$. Finally, using the lemma, we see that

(3) $\alpha_{n}+B_{n-1}<B_{n-1}+\alpha_{n} \leq B_{n-1}+C_{1}<B_{n-1}+C_{2}<\ldots<$

$$
B_{n-1}+C_{m-1}<B_{n-1}+C_{n}
$$

so that by (2) and (3),

$$
\begin{gathered}
\alpha_{n}+B_{m}<\alpha_{n}+B_{m+1}<\ldots<\alpha_{n}+B_{n-1}<B_{n-1}+C_{1} \\
\quad<B_{n-1}+C_{2}<\ldots<B_{n-1}+C_{m-1}<B_{n-1}+C_{n} .
\end{gathered}
$$

This proves that $\alpha_{n}+B_{i}, i=m, m+1, \ldots, n-1$, together with $B_{n-1}+C_{j}$, $j=1,2, \ldots, m-1, n$, are $n$ distinct sums for $\alpha_{1}, \alpha_{2}, \ldots, \alpha_{n}$.

For all $n \geq 1, \omega+1, \omega+2, \ldots, \omega+n$ are $n$ pairwise-noncommutative ordinals with sums $\omega n+1, \omega n+2, \ldots, \omega n+n$. Thus, $\min P_{n}=n$. Finally, Wakulicz [3] has shown, in effect, that the maximal sum, $m_{n}$, for $E_{n}$ can always be obtained by using $n$ pairwise-noncommutative ordinals of the form

$$
\begin{gathered}
\omega^{2 r} ; \omega^{2 r-1}+\omega^{2 r-2}, \omega^{2 r-1} \cdot 2+\omega^{2 r-2} \cdot 2, \omega^{2 r-1} \cdot 4+\omega^{2 r-2} \cdot 3, \\
\ldots, \omega^{2 r-1} \cdot 2^{x_{r}-1}+\omega^{2 r-2} \cdot x_{r} ; \\
\omega^{2 r-3}+\omega^{2 r-4}, \omega^{2 r-3} \cdot 2+\omega^{2 r-4} \cdot 2, \omega^{2 r-3} \cdot 4+\omega^{2 r-4} \cdot 3, \\
\ldots, \omega^{2 r-3} \cdot 2^{x_{r-1}-1}+\omega^{2 r-4} \cdot x_{r-1} \\
\vdots \\
\omega+1, \omega 2+2, \omega 4+3, \ldots, \omega 2^{x_{1}-1}+x_{1},
\end{gathered}
$$

where $x_{1}+x_{2}+\ldots+x_{r}=n-1$. 
Corollary $P_{1}=\{1\}=E_{1}$ and $P_{2}=\{2\}=E_{2}-\{1\}$.

Theorem 3 Let $n \geq 2$. Then the following integers are in $P_{n}$ :

(a) $n, n+1, \ldots, 2 n-2$

(b) For $n \geq 3$ and for $1 \leq \ell \leq n-2$, all integers of the form $(n-2)^{2}+\ell(n-$ 2) +2

(c) For $n \geq 4, n(n-1)$

(d) For $n \geq 5, n^{2}-2$

(e) $2^{n-1}$

(f) $n^{2}-3 n+3$

(g) $n^{2}-3 n+4$.

Proof: Unless otherwise indicated, assume $n \geq 2$.

(a) For $1 \leq \ell \leq n-1$, the $n$ pairwise-noncommutative ordinals $\omega+1$, $\omega+2, \ldots, \omega+(n-1)$, and $\ell$ have sums

$$
\omega(n-1)+1, \omega(n-1)+2, \ldots, \omega(n-1)+n-1+\ell .
$$

Thus $\{n, n+1, \ldots, 2 n-2\} \subseteq P_{n}$.

(b) Let $n \geq 3$ and let $1 \leq \ell \leq n-2$. Then $\omega^{2}, \omega+1, \omega+2, \ldots, \omega+(n-$ 2 ), and $\ell$ have sums $\omega^{2}, \omega^{2}+\ell ; \omega^{2} \cdot i+j$, where $1 \leq i \leq n-2$ and $1 \leq j \leq n-$ $2+\ell$. Consequently, for each such $\ell$ there are $(n-2)^{2}+\ell(n-2)+2$ distinct sums.

(c) For $n \geq 4$, the ordinals $\omega^{2} ; \omega 2+1, \omega+2, \omega+3, \omega+(n-1)$ yield the $n(n-1)$ distinct sums $\omega^{2} ; \omega^{2}+\omega+j$, for $2 \leq j \leq n-1 ; \omega^{2}+\omega i+j$, for $2 \leq$ $i \leq n$ and $1 \leq j \leq n-1$.

(d) For $n \geq 5$, the ordinals $\omega^{2}, \omega 3+1, \omega+2, \omega+3, \ldots, \omega+(n-1)$ have as sums $\omega^{2} ; \omega^{2}+\omega i+j$, for $i=1,2$ and $2 \leq j \leq n-1 ; \omega^{2}+\omega i+j$, for $3 \leq$ $i \leq n+1$ and $1 \leq j \leq n-1$. Thus there are $(n-1)^{2}+2(n-2)+1$, or $n^{2}-$ 2 distinct sums.

(e) The ordinals $\omega^{n-1}, \omega^{n-2}, \ldots, \omega^{2}, \omega, 1$ yield $2^{n-1}$ distinct sums.

(f) The ordinals $\omega^{2}, \omega^{2}+\omega, \omega, \omega+1, \omega+2, \ldots, \omega+(n-3)$ have as sums $\omega^{2} \cdot 2 ; \omega^{2} \cdot 2+\omega i+j$, for $1 \leq i \leq n-1$ and $0 \leq j \leq n-3$. There are $n^{2}-$ $3 n+3$ distinct sums.

(g) The ordinals $\omega^{2}, \omega+2, \omega+4, \ldots, \omega+2(n-2), 2$ have as sums $\omega^{2}$, $\omega^{2}+2 ; \omega^{2}+\omega i+2 j$, for $1 \leq i \leq n-2$ and $1 \leq j \leq n-1$. There are $n^{2}-3 n+$ 4 distinct sums.

Theorem $4 \quad P_{3}=\{3,4,5\}=E_{3}-\{1,2\}$.

Proof: $P_{3} \subseteq E_{3}=\{3,4,5\}$. Moreover $\{3,5\} \subseteq P_{3}$ by Theorem 2 and $4 \in P_{3}$ by part (a) of Theorem 3.

Lemma 2 In order for 4 ordinals to yield 11 or more different sums, one of these must have highest degree and the other three must have the same degree.

Proof: Given any 4 ordinals, let $\delta$ be the highest degree of any of these. Then it is easily seen that if all 4 ordinals are of degree $\delta$, there are at most 4 different sums; if 3 of the ordinals are of degree $\delta$, there are at most 6 different sums; if 2 of the ordinals are of degree $\delta$ there are at most 10 different sums. 
Now suppose that exactly one of the ordinals, $\alpha_{\delta}$, is of degree $\delta$. Let $\gamma$ be the highest degree among the other 3 ordinals. If exactly one ordinal, $\alpha_{\gamma}$, is of degree $\gamma$ and if $\beta_{1}$ and $\beta_{2}$ are the remaining ordinals, there are at most 10 possible sums: $\alpha_{\delta}, \alpha_{\delta}+\beta_{1}, \alpha_{\delta}+\beta_{2}, \alpha_{\delta}+\beta_{1}+\beta_{2}, \alpha_{\delta}+\beta_{2}+\beta_{1}, \alpha_{\delta}+\alpha_{\gamma}, \alpha_{\delta}+$ $\alpha_{\gamma}+\beta_{1}, \alpha_{\delta}+\alpha_{\gamma}+\beta_{2}, \alpha_{\delta}+\alpha_{\gamma}+\beta_{1}+\beta_{2}$, and $\alpha_{\delta}+\alpha_{\gamma}+\beta_{2}+\beta_{1}$. If exactly two ordinals, $\alpha_{1}$ and $\alpha_{2}$ are of degree $\gamma$ and if $\beta$ is the remaining ordinal, there are at most 10 possible sums: $\alpha_{\delta}, \alpha_{\delta}+\beta, \alpha_{\delta}+\alpha_{1}, \alpha_{\delta}+\alpha_{2}, \alpha_{\delta}+\alpha_{1}+\alpha_{2}, \alpha_{\delta}+$ $\alpha_{2}+\alpha_{1}, \alpha_{\delta}+\alpha_{1}+\beta, \alpha_{\delta}+\alpha_{2}+\beta, \alpha_{\delta}+\alpha_{1}+\alpha_{2}+\beta$, and $\alpha_{\delta}+\alpha_{2}+\alpha_{1}+\beta$. The lemma is thereby established.

\section{Lemma 34 pairwise noncommutative ordinals cannot yield 11 sums.}

Proof: By Lemma 2, it suffices to consider ordinals with remainder form $\omega^{\gamma} \ell_{i}+$ $\rho_{i}$ for $i=1,2$, and 3 together with $\alpha$, where $\operatorname{deg}(\alpha)>\gamma$. Clearly, because the ordinals are pairwise-noncommutative, $\rho_{1}, \rho_{2}$, and $\rho_{3}$ are distinct. We can assume $\ell_{1} \leq \ell_{2} \leq \ell_{3}$. The possible sums for these ordinals are then

$$
\begin{aligned}
& \beta_{1}=\alpha \\
& \beta_{2}=\alpha+\omega^{\gamma} \ell_{1}+\rho_{1} \\
& \beta_{3}=\alpha+\omega^{\gamma} \ell_{2}+\rho_{2} \\
& \beta_{4}=\alpha+\omega^{\gamma} \ell_{3}+\rho_{3} \\
& \beta_{5}=\alpha+\omega^{\gamma}\left(\ell_{1}+\ell_{2}\right)+\rho_{1} \\
& \beta_{6}=\alpha+\omega^{\gamma}\left(\ell_{1}+\ell_{2}\right)+\rho_{2} \\
& \beta_{7}=\alpha+\omega^{\gamma}\left(\ell_{1}+\ell_{3}\right)+\rho_{1} \\
& \beta_{8}=\alpha+\omega^{\gamma}\left(\ell_{1}+\ell_{3}\right)+\rho_{3} \\
& \beta_{9}=\alpha+\omega^{\gamma}\left(\ell_{2}+\ell_{3}\right)+\rho_{2} \\
& \beta_{10}=\alpha+\omega^{\gamma}\left(\ell_{2}+\ell_{3}\right)+\rho_{3} \\
& \beta_{11}=\alpha+\omega^{\gamma}\left(\ell_{1}+\ell_{2}+\ell_{3}\right)+\rho_{1} \\
& \beta_{12}=\alpha+\omega^{\gamma}\left(\ell_{1}+\ell_{2}+\ell_{3}\right)+\rho_{2} \\
& \beta_{13}=\alpha+\omega^{\gamma}\left(\ell_{1}+\ell_{2}+\ell_{3}\right)+\rho_{3}
\end{aligned}
$$

Some of these 13 sums may be the same.

If $\ell_{1}=\ell_{2}=\ell_{3}$, then $\beta_{5}=\beta_{7}, \beta_{6}=\beta_{9}$, and $\beta_{8}=\beta_{10}$, so that there are 10 distinct sums.

If $\ell_{1}=\ell_{2}<\ell_{3}$, then $\beta_{8}=\beta_{10}$, and there are 12 distinct sums.

If $\ell_{1}<\ell_{2}=\ell_{3}$, then $\beta_{5}=\beta_{7}$, and there are 12 distinct sums.

If $\ell_{1}<\ell_{2}<\ell_{3}$, then there are 13 distinct sums.

$$
\text { Theorem } 5 \quad \begin{aligned}
P_{4} & =\{4,5,6,7,8,9,10,12,13\} \\
& =E_{4}-\{1,2,3,11\} .
\end{aligned}
$$

Proof: By [5] together with Theorem 2 and Lemma 3 of this paper, $P_{4} \subseteq\{4,5$, $6,7,8,9,10,12,13\}$. Moreover, $\{4,13\} \subseteq P_{4}$ by Theorem $2,\{5,6\} \subseteq P_{4}$ by Theorem 3 (a), $\{8,10\} \subseteq P_{4}$ by Theorem 3 (b), $12 \in P_{4}$ by Theorem 3 (c), and $7 \in P_{4}$ by Theorem 3 (f). Finally, the ordinals $\omega^{2}, \omega, \omega 2+1$, and 1 have 9 distinct sums: $\omega^{2}, \omega^{2}+1, \omega^{2}+\omega, \omega^{2}+\omega+1, \omega^{2}+\omega 2+1, \omega^{2}+\omega 2+2, \omega^{2}+\omega 3$, $\omega^{2}+\omega 3+1$, and $\omega^{2}+\omega 3+2$.

The cases of 5 and 6 pairwise-noncommutative ordinals will be considered in [8]. 


\section{REFERENCES}

[1] Erdös, P., "Some remarks on set theory," Proceedings of the American Mathematical Society, vol. 1 (1950), pp. 127-141.

[2] Sierpiński, W., "Sur les series infinies de nombres ordinaux," Fundamenta Mathematicae, vol. 36 (1949), pp. 248-253.

[3] Wakulicz, A., "Sur la somme d'un nombre fini de nombres ordinaux," Fundamenta Mathematicae, vol. 36 (1949), pp. 254-266.

[4] Wakulicz, A., "Correction au trauvail "Sur les sommes d'un nombres fini de nombres ordinaux" de A. Wakulicz," Fundamenta Mathematicae, vol. 38 (1951), p. 239.

[5] Wakulicz, A., "Sur les sommes de quatre nombres ordinaux," Polska Akademia Umiejetności, Sprawozdania z Czynności i Posiedzén, vol. 42 (1952), pp. 23-28.

[6] Zuckerman, M., "Sums of at most 8 ordinals," Zeitschrift für Mathematische Logik und Grundlagen der Mathematik, vol. 19 (1973), pp. 435-446.

[7] Zuckerman, M., "Sums of at least 9 ordinals," Notre Dame Journal of Formal Logic, vol. 14 (1973), pp. 263-268.

[8] Zuckerman, M., "Sums of 5 or 6 pairwise-noncommutative ordinals," to appear in Zeitschrift für Mathematische Logik und Grundlagen der Mathematik.

City College of the City University of New York

New York, NY 10025 